\title{
Interférences
}

Ars scribendi

$10 \mid 2018$

Varia

\section{Divinité et espoir millénariste au temps des guerres civiles : pour une relecture de Virgile et d'Horace}

\section{Aliénor Cartoux}

\section{(2) OpenEdition}

\section{Journals}

Édition électronique

URL : http://journals.openedition.org/interferences/5990

DOI : 10.4000/interferences.5990

ISSN : $1777-5485$

Éditeur

HiSoMA - Histoire et sources des Mondes antiques

\section{Référence électronique}

Aliénor Cartoux, «Divinité et espoir millénariste au temps des guerres civiles : pour une relecture de Virgile et d'Horace », Interférences [En ligne], 10 | 2018, mis en ligne le 26 juillet 2018, consulté le 15 septembre 2020. URL : http://journals.openedition.org/interferences/5990 ; DOI : https://doi.org/ 10.4000/interferences.5990

Ce document a été généré automatiquement le 15 septembre 2020.

Tous droits réservés 


\title{
Divinité et espoir millénariste au temps des guerres civiles : pour une relecture de Virgile et d'Horace
}

\author{
Aliénor Cartoux
}

\section{Introduction}

Dico te, deus noster, omnis creaturae creatorem, «J'affirme que c'est toi, notre Dieu, qui es le créateur de toute créature » (Augustin, Conf.,

$\mathrm{XI}, 12)$.

1 À l'heure du christianisme, saint Augustin peut parler sans équivoque du Deus : son lecteur y reconnaît immédiatement le Dieu unique, celui du christianisme. Mais lorsqu'un auteur païen mentionne simplement un deus, il arrive que le référé ne puisse être clairement identifié, faute de mention précise d'un nom. C'est sur la richesse de cette ambiguïté que nous souhaiterions porter notre attention.

2 J. Griffin a montré la nécessité d'une analyse diachronique de certains thèmes élaborés dans la littérature antique, dont fait partie, selon lui, la représentation du culte ${ }^{1}$. C'est à l'identification littéraire des hommes politiques du second triumvirat avec un héros ou un dieu salvateur ${ }^{2}$ que nous souhaiterions nous intéresser, par une étude à la fois diachronique et synchronique. Les travaux de J. Scheid nous permettent désormais d'éviter un écueil fondamental: celui de s'interroger sur la notion de croyance concernant la religion romaine ${ }^{3}$. Réfléchir à la possibilité que les Romains aient véritablement cru à la divinité de certains hommes n'a pas de sens. Ce qui fait qu'un dieu est un dieu, ce sont les cultes qui lui sont rendus ${ }^{4}$, ce qui implique de prendre en compte les variantes cultuelles des différents espaces de célébration.

3 À l'époque triumvirale, il n'y a, et il ne peut y avoir, de culte de la personne, à la différence de ce qui se mettra en place avec le culte impérial. Les critiques modernes ont pourtant mis en lumière l'utilisation omniprésente de la religion à des fins 
personnelles durant les guerres civiles de la fin de la République ${ }^{5}$, première étape de ce qui sera un jour le culte impérial ${ }^{6}$. À la fin des années 40 av. J.-C., l'heure est aux espérances: la bataille de Modène a permis d'éliminer les opposants aux futurs triumvirs; la guerre de Pérouse, quant à elle, a mis fin à la révolte qui s'élevait autour de Lucius Antonius, et a ainsi laissé le champ libre à Octavien en Italie. La tension qui aurait pu se raviver entre ce dernier et Antoine, du fait notamment de la répression contre Lucius Antonius, parent d'Antoine, n'est plus d'actualité en cette fin de l'année 40 av. J.-C., grâce à la paix de Brindes ${ }^{7}$. Mais, des années 44 à 40 av. J.-C., la lutte fut acharnée, et la guerre fut aussi une guerre de « communication ». Comme le note P. Jal, « en vertu à la fois de sa nature et des conditions dans lesquelles les [...] adversaires la firent au $\mathrm{I}^{\mathrm{er}}$ siècle avant J.-C., la guerre civile exigeait dans chaque camp cet appel à l'aide divine ${ }^{8}$ ». La religion fut donc au cœur d'une véritable propagande des différents partis politiques en lutte?

4 La question de la pertinence de la notion de propagande a été bien posée et analysée par P. Le Doze ${ }^{10}$. Pour notre part, nous ne sommes pas certaine que le terme implique nécessairement un système cohérent de pensée destiné à manipuler les masses, ce que P. Le Doze appelle une "idéologie $»^{11}$. Nous définirons donc ici la "propagande» comme la mise en place de différents procédés afin de diffuser, le plus largement possible, une image possédant certains traits spécifiques et caractéristiques, et censée appuyer une idée, une personne, une institution ou un type de gouvernement, à destination d'un public ciblé. L'un de ces "procédés " potentiels est la littérature. Selon P. Jal, «la guerre civile introduit [...] dans la propagande religieuse un élément beaucoup plus caractéristique encore : il s'agit du lien qui s'établit entre chaque parti et une divinité particulière", une véritable "annexion de telle ou telle divinité par chacun des deux camps $»^{12}$. Il nous semble que la représentation littéraire, et avant tout poétique, fourmille d'indications précieuses, étant elle-même une autre «fabrique des dieux ", non pas civile, mais mythique, selon la tripartition varronienne ${ }^{13}$. Puisqu'il n'y a pas de divinisation de la personne, au sens cultuel du terme, c'est à la place qu'a pu prendre la littérature dans cette guerre de revendication divine que nous nous intéresserons. L'expression d'une relation privilégiée entre certains hommes d'exception et une divinité particulière y bat son plein à cette époque. Quel rôle la littérature latine, tout imprégnée qu'elle est alors de la culture hellénistique ${ }^{14}$ - qui ne craint pas la divinisation d'hommes politiques vivants -, joue-t-elle par rapport à cette guerre de communication durant l'époque triumvirale?

\section{Les Bucoliques I et IV (40-39 av. J.-C.) : de la divinisation circonstanciée à la divinité indéterminée}

I. Gradel, dans un ouvrage récent, étudie minutieusement les différents termes pouvant renvoyer à la divinité, dans ses diverses acceptions. Le terme deus est sans doute celui qui semble le moins ambigu dans sa signification : il désigne un dieu, considéré comme tel dans toutes les caractéristiques de son être ${ }^{15}$. Pourtant, son utilisation par Virgile dans la première Églogue ${ }^{16}$ pour désigner allusivement la figure montante d'Octavien, à la fin des années $40 \mathrm{av}$. J.-C., sert non pas à clarifier son statut, mais bien à créer ce que P. Heuzé appelle une « fausse évidence ${ }^{17}$ » (Virgile, Ecl., I, 6-8) : 
O Meliboee, deus nobis haec otia fecit :

namque erit ille mihi semper deus ; illius aram

saepe tener nostris ab ouilibus imbuet agnus.

«Mélibée, c'est un dieu qui nous a octroyé ces loisirs : pour moi il sera toujours un

dieu ; c'est son autel qu'un tendre agneau tiré de nos bergeries ensanglantera. »

Que Virgile retravaille ou non par la fiction poétique une expérience personnelle ${ }^{18}$, la référence historique reste unanimement identifiée ${ }^{19}$ : il s'agit d'une allusion aux expropriations de l'année 40 av. J.-C. Le deus dont la puissance permet la conservation des terres servirait donc de référence à Octavien, pour son rôle dans cet événement historique. Mais pourquoi l'usage d'une telle dénomination ${ }^{20}$ ? Un glissement intéressant s'opère à la fin du vers $7:$ la double mention de deus semble être justifiée par l'apparition d'aram en fin de vers. L'autel dont parle Tityre est métaphorique, et n'existe que dans l'imaginaire du personnage et du poète. Faut-il n'y voir, comme cela a souvent été le cas, qu'une expression hyperbolique héritée de la poésie hellénistique ${ }^{21}$ ? Cette interprétation nous semble à tout le moins insuffisante pour expliquer le choix particulier de Virgile en cet endroit de son œuvre, et surtout l'absence de dénomination explicite d'Octavien. D'autres réponses peuvent être avancées: la volonté d'établir, dès les premiers vers, une connivence intellectuelle et culturelle avec le lecteur contemporain, celle de ne pas rompre la fiction diégétique, enfin celle de ne pas donner l'impression de céder ouvertement à la poésie de cour. Toutes ces explications sont valables, mais insuffisantes prises indépendamment. Selon nous, c'est par l'analyse de la IV Églogue, rédigée peu de temps avant, que le débat peut être éclairé et enrichi.

7 Virgile, dans cette Bucolique, joue une nouvelle fois de la connivence avec son lecteur pour lui suggérer l'identité du sujet de son poème, le puer divin ${ }^{22}$ qu'il ne nomme jamais. Il convient de prendre en compte le contexte fictionnel des deux Églogues car, nous le verrons, il détermine la "poétique de l'énigme ${ }^{23}$ » mise en place par Virgile: tandis que, dans la première Bucolique, Virgile retravaille le matériau historique pour le fondre entièrement dans la fiction bucolique, dans la $\mathrm{IV}^{\mathrm{e}}$ Bucolique il réactualise le mythe de l'âge d'or ${ }^{24}$ pour l'insérer dans un contexte historique, le sien ${ }^{25}$.

8 Là encore, l'identification du deus à Octavien est séduisante ${ }^{26}$. Pourtant, plusieurs éléments résistent à cette interprétation, au premier rang desquels se trouve le jeune âge de cette figure salvatrice. Bien d'autres hypothèses d'interprétation ont été suggérées $^{27}$, et c'est justement cette abondance d'analyses qui les fragilise, à la fois dans leur visée et dans leurs conclusions. J.-P. Brisson avait lui-même fait ce constat : «On s'est perdu, et on se perd en conjectures pour identifier ce mystérieux héros ${ }^{28} »$. Il relève donc le vice que comporte ontologiquement toute analyse cherchant un renvoi au réel sous la représentation littéraire. Mais, de ce refus initial de découvrir la réalité masquée derrière la représentation littéraire, il finit cependant, quelques pages plus loin, par succomber à la tentation : «Et si cet enfant, à la naissance de qui le poète en quelque sorte assiste, n'était autre qu'une représentation du poème lui-même ${ }^{29}$ ? " J.-P. Brisson, voulant se garder des écueils qu'il a soulignés, ne peut cependant s'empêcher de voir dans ce poème une dimension auto-réflexive, le regard du poète sur la naissance de son propre poème. Mais cette identification n'est pas parfaite, et son auteur en souligne lui-même les limites en affirmant que la fin de l'Églogue, qui présente l'enfant souriant à sa mère, ne peut s'accorder avec son analyse métalittéraire. Il tente alors de sortir de l'aporie en affirmant que «la naissance et la croissance de cet 'enfant' sont des apparences trompeuses qui masquent d'autres sens ${ }^{30}$ 
", comme si Virgile voulait que l'identification fût, non pas difficile et complexe, mais imparfaite. C'est alors que le critique propose une seconde interprétation, orphique, pouvant éclairer la présence et les implicites de cet enfant providentiel, qui serait alors Dionysos, comme pour pallier les déficiences de la première ${ }^{31}$.

Si nous nous sommes attardée sur l'interprétation de J.-P. Brisson, ce n'est pas parce qu'elle nous semble plus fautive qu'une autre. Toutes les analyses qui ont été faites ont, selon nous, leur part de pertinence, mais aussi leur part d'imperfection; celle que nous avons précédemment défendue ${ }^{32}$, qui voudrait voir dans ce puer une image, reconstruite selon des codes littéraires, de la figure montante d'Octavien, a elle aussi ses insuffisances. Le développement de J.-P. Brisson a retenu notre attention car il est à l'origine d'une hypothèse que nous souhaiterions développer et défendre ${ }^{33}$ : si aucune identification ne se suffit à elle-même, et que chacune tend à en appeler une autre, c'est que toutes sont justes et incomplètes en même temps, qu'aucune ne peut être privilégiée au point d'effacer toutes les autres. L'effet voulu par Virgile est alors proprement littéraire, en ce qu'il peut jouer sur les différents niveaux d'interprétation et de renvoi au réel, par son ambiguïté et sa complexité. Le puer virgilien est un produit littéraire, dont l'identification parfaite et donc ultime au réel est impossible. Il est à la fois et autant le poème naissant de Virgile, le Dionysos orphique, le jeune Octave ou encore son rival politique, Antoine. Cette incapacité à fixer une identification nette et précise est, selon nous, le but visé par Virgile dans la représentation de la figure

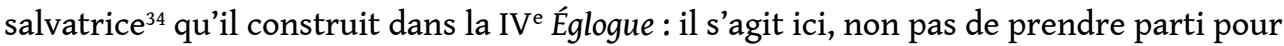
une instance qui, selon lui, serait la seule permettant à Rome de retrouver l'âge d'or mythique et rêvé par ses contemporains, mais simplement de traduire cet espoir millénariste de l'époque, et son incertitude quant à l'entité capable d'assumer cette fonction ${ }^{35}$. Virgile adresse, à ceux qui s'en sentiraient la capacité, un appel à endosser ce rôle salvateur. Nous ne pouvons que trop rappeler l'importance ici de ne pas faire de projection rétrospective. En 40 av.J.-C., date de rédaction de la IV Églogue, de nombreux espoirs animaient la population de l'Empire romain: la paix de Brindes venait d'être signée et promettait un temps meilleur, aussi bien grâce à la personne d'Octavien qu'à celle d'Antoine. La propagande triumvirale de l'époque laisse bien percevoir la prétention divine de chacun des hommes politiques du jour ${ }^{36}$. Aussi, la correspondance que nous pouvons établir entre ce rôle littéraire et celui qu'Auguste prétendit par la suite avoir rempli doit-elle être revue conformément à la réception immédiate du poème. Si, aujourd'hui, l'interprétation la plus séduisante du poème nous semble être une identification précoce d'Octavien avec le héros salvateur, c'est parce qu'a posteriori Auguste a tenté de correspondre à cette image proprement littéraire ${ }^{37}$. La littérature explicite donc ce qui n'est qu'en germe dans les mentalités et les événements de l'époque ${ }^{38}$.

Dans les deux Églogues, de manière adaptée au contexte fictionnel de chaque poème, Virgile laisse donc place à la traduction des potentialités de son temps : Octavien, figure émergente de l'époque, peut prétendre aux honneurs divins, mais leur attribution est encore sujette à condition, et l'échec n'est jamais loin, ce que suggèrent les restrictions à l'identification présentes dans les deux poèmes. L'interprétation qui ferait d'Octavien un dieu par les bienfaits qu'il apporterait à son peuple ${ }^{39}$ n'est que l'une des possibilités, que seul le déroulement historique de l'époque pourra confirmer ou infirmer. Virgile joue ainsi des codes et des potentialités offertes par les différents niveaux de lecture de 
son œuvre pour exprimer au plus près la situation et les attentes de son époque, auxquelles il donne voix.

11 Virgile n'est d'ailleurs pas le seul poète à s'être interrogé sur l'avenir que pouvaient offrir les hommes politiques de son temps. Le dialogue instauré entre Virgile et Horace à cette époque a depuis longtemps été relevé ; ainsi W. Fitzgerald note que, « par-dessus tout, c'est la question du rôle du poète lors d'une période de bouleversement politique qui fait des Épodes un successeur des Églogues ${ }^{40} »$.

\section{L'Épode XVI ${ }^{41}$ d'Horace (39 av. J.-C.) ${ }^{42}$ : le retrait dans un mythe déserté par les dieux}

12 L'un des premiers poèmes "civiques» de l'époque des guerres civiles, l'Épode XVI d'Horace, est adressé ad populum Romanum; la dédicace annonce immédiatement la dimension collective que la voix du poète endosse dans cette épode ${ }^{43}$.

Les vers 1 à 14 énumèrent, après un brutal rappel de la guerre intestine, les grands combats historiques dont les citoyens romains sont ressortis victorieux, pour finir sur l'image saisissante du créateur de la race, Romulus. On passe donc de l'actualité immédiate au passé historique, puis au passé mythique, paradoxalement réactualisé par le futur dissipabit ${ }^{44}$. C'est cet éloignement progressif, ce refus du présent permis par l'échappée vers le mythe, que prolonge Horace à l'échelle macro-structurale. Les vers 15 à 41 introduisent explicitement la volonté d'une fuite de Rome, et avec elle, de son présent, et s'achèvent sur une série d'adunata qui signifient le départ définitif et irrémédiable de Rome. Dans un troisième mouvement, les vers 42 à 66 présentent le nouveau séjour des Bienheureux, reprenant les traits topiques du mythe de l'âge d'or ${ }^{45}$, pour l'opposer au monde dans lequel les Romains vivent actuellement, l'âge de fer. À la détresse des Romains, la réponse qu'apporte Horace est sans appel et dépourvue d'espérance.

Ce qui nous semble particulièrement notable dans ce poème est l'absence quasi totale des dieux dans l'environnement mythique de l'âge d'or, dont l'une des caractéristiques principales est pourtant l'entente harmonieuse entre hommes et divinités ${ }^{46}$. Certes, Cérès et Jupiter apparaissent une fois dans ce monde mythique ${ }^{47}$; mais ils sont ici l'allégorie de la nature bien plus que de véritables divinités. Seul Jupiter est évoqué sous sa forme anthropomorphisée au vers 63, mais comme responsable de la dégradation des âges et de la préservation de l'île des Bienheureux, où il ne séjourne pourtant pas lui-même. Cette omission, la seule parmi tous les topoi du mythe, ne peut être attribuée qu'à un choix délibéré. Elle nous semble pouvoir être interprétée comme la réponse d'Horace à la monopolisation des dieux par les triumvirs et à l'espoir optimiste de la IVe Églogue : des chefs s'assimilant à des dieux, il ne faut rien attendre. Selon lui, il n'existe rien de tel qu'un héros ou un dieu salvateur. À la propagande religieuse des politiques, Horace oppose sa voix de uates comme seul recours salvateur possible ${ }^{48}$. Si l'interprétation du vers 66 est encore controversée dans son rapport au sens général du poème ${ }^{49}$, le terme uates reste néanmoins empreint de connotations prophétiques et civiles fortes. Horace réintroduirait donc la figure du poète sur la scène politique. Seul le poète pourra faire retrouver la pietas à son peuple, l'unique vertu capable de rendre au peuple romain un âge d'or digne des îles des Bienheureux ${ }^{50}$. 
Nous proposons donc de lire l'Épode XVI d'Horace et l'Églogue IV de Virgile dans un rapport dialogique fait d'opposition mais aussi de complémentarité. Si les deux poèmes font un large usage du mythe, ils lui accordent deux fonctions distinctes : pour Virgile, le mythe est une promesse d'avenir; pour Horace, il est une échappée du présent ${ }^{51}$. Dans les deux poèmes cependant, le traitement du mythe permet de faire émerger la voix de l'auteur sur la scène politique. De ce point de vue, les Géorgiques sont les héritières parfaites tant des Bucoliques que des Épodes.

\section{Les Géorgiques : divinisation poétique et gloire politique}

En 38 avant J.-C. ${ }^{52}$, Virgile construit une nouvelle vision de l'âge d'or au début des Géorgiques $^{53}$, en s'attachant à la transition entre l'âge d'or et l'âge de fer. La modification la plus innovante eu égard à la tradition mythologique antérieure, c'est avant tout, comme le note M. O. Lee ${ }^{54}$, que la transition entre les deux âges n'est pas perçue comme une déchéance à regretter, un paradis perdu, mais au contraire comme une source de progrès pour l'homme $\mathrm{e}^{55}$. Pourquoi un tel revirement par rapport à l'Églogue IV, et une telle modification du mythe? Ne faut-il y voir qu'une adaptation au contenu didactique de l'œuvre et aux codes littéraires du genre?

Sans doute Virgile a-t-il tenu compte de la réponse que lui offrait Horace dans son Épode; sans doute aussi les événements ne lui permettaient-ils plus d'espérer le retour d'un tel âge d'or ${ }^{56}$ : la paix de Brindes ne fut qu'une trêve bien courte, tout comme la paix de Misène, qui laissa place à de nouvelles guerres. Mais c'est la dynamique d'ensemble du chant I des Géorgiques qui, selon nous, permet de comprendre les modifications apportées par Virgile au mythe de l'âge d'or. O.M. Lee a souligné l'encadrement du poème par deux prières d'invocation aux divinités, qui impliquent Octavien ${ }^{57}:$ "Virgile achève le livre I comme il l'a commencé, avec une prière pour que les dieux de son pays épargnent le jeune Octavien, qui arrive en soter, en sauveur, pour une Rome en proie aux troubles. [...] En définitive, tandis que le livre I est un portrait classique de l'homme luttant contre la nature depuis ses tout premiers jours, forçant la terre à être productive et, au cours de ses efforts, se transformant en l'animal le plus créatif, il peut aussi être considéré comme le portrait, au travers de la métaphore de la nature, d'un homme prodigieux luttant pour ramener de l'ordre dans le chaos de l'histoire, d'un homme chargé d'une mission évolutionniste, sur le chemin de sa divinisation. C'est pourquoi, à l'ouverture et à la fermeture des descriptions de son œuvre, on trouve une prière ${ }^{58} \%$. Nous pensons que c'est la place qu'il accorde à Octavien dans son œuvre, l'espoir qu'il fonde sur lui, qui pousse Virgile à modifier son mythe : l'âge d'or est un temps révolu ; les guerres civiles ont duré trop longtemps pour qu'on puisse espérer un retour. Mais, si les temps à venir seront laborieux, ils permettront pourtant aux Romains d'œuvrer pour tirer les leçons de leurs erreurs, et pour construire un monde meilleur, toujours en quête de progrès, si tant est qu'un bon gouvernant les y aide. Dans les deux prières du livre I, Octavien est bien promis à la divinisation ${ }^{59}$, conformément au rôle de héros salvateur que le poète l'invite à endosser.

Pour mieux comprendre la divinisation que promet Virgile à Octavien, il faut nous tourner vers le début du chant $\mathrm{III}^{60}$. Il s'agit du célèbre passage dans lequel Virgile 
promet à Octavien d'élever un temple en son honneur. F. Della Corte ${ }^{61}$ considère qu'imaginer la statue d'Octavien dans un temple alors qu'il n'est pas encore mort marque un premier pas dans le long processus qui conduira à la divinisation d'Auguste. Mais nous pensons que la véritable divinisation dont il est ici question est la divinisation littéraire, et non l'anticipation sur la théologie civile. On a souvent considéré que le templum que Virgile souhaitait construire, et qui serait à la gloire d'Octavien, était une annonce de l'Énéide ${ }^{62}$. Mais, comme le rappelle O. M. Lee, l'épopée qui naîtra dix ans plus tard suivra une trame bien différente de celle développée ici ${ }^{63}$. Nous préférons pour notre part y voir une parole performative: le templum qui est présenté comme étant à venir, c'est en fait la description littéraire de ce temple qu'en fait hic et nunc Virgile ${ }^{64}$; le futur n'est qu'une manière de suggérer à Octavien que la divinisation ne pourra vraiment prendre effet que s'il est à la hauteur des espérances que le poète place en lui. Si la littérature permet une divinisation, c'est bien celle de la mémoire ; les générations à venir se souviendront de l'hommage que lui a rendu Virgile uniquement s'il mène à bien son entreprise. Dans cette perspective, un glissement sémantique s'opère : la couronne d'olivier n'est plus celle du vainqueur mais du prêtre, et les dona ne sont plus les prix du concours mais les offrandes ${ }^{65}$. Ou plutôt, elles sont les deux : car si Virgile se présente comme prêtre d'Octavien, prêt à servir son culte s'il s'en montre digne, il est, lui aussi, un uictor ${ }^{66}$, qui mérite les récompenses de la victoire $^{67}$. Le sort $d u$ politique et celui du poète uates sont donc dans une relation d'interdépendance ${ }^{68}$ : seul le poète peut offrir à l'imperator la divinisation qu'il mérite, et, ce faisant, la même gloire éternelle rejaillit sur lui ${ }^{69}$. On ne peut faire plus forte revendication du pouvoir de la poésie.

\section{Conclusion}

19 À l'heure du triumvirat et de ses guerres civiles, l'incertitude règne, et les poètes sont pour nous les témoins précieux de ce temps d'indécision, entre angoisse et espoir, auquel ils ont donné une traduction littéraire, en même temps qu'ils ont pensé le rôle du poète et de la poésie en ces temps troublés de différentes manières. Il est significatif que le mythe choisi par Virgile comme par Horace soit celui de l'âge d'or : s'il signifie bien l'avènement d'une époque bienheureuse, il possède comme composante essentielle l'harmonie entre hommes et dieux à l'heure où les guerres civiles sont ressenties comme une rupture de la pax deorum. C'est aussi cette réflexion sur le divin, sur la capacité des triumvirs à endosser ce rôle providentiel valorisée dans leur propagande, qui unit la production des deux poètes.

20 Il serait bénéfique, à notre avis, d'approfondir une réflexion que nous ne pouvons que suggérer ici : celle de l'héritage poétique triumviral, tel que nous l'avons envisagé, dans les Métamorphoses d'Ovide ${ }^{70}$. Le constat est immédiat dès le livre I des Métamorphoses, qui s'ouvre sur une cosmogonie ${ }^{71}$ orchestrée par un deus $^{72}$ à l'identité mystérieuse, comme dans la première Églogue, et se poursuit avec une retractatio du mythe de l'âge d'or et du passage à l'âge de fer ${ }^{73}$, provoqué par Jupiter ${ }^{74}$, où, comme dans l'Épode XVI, il convient de noter l'absence du motif de l'harmonie entre hommes et dieux. Certes, à l'inverse de la première Églogue, la nature divine du mystérieux deus, mundi fabricator ${ }^{75}$, est cette fois annoncée par la voix poétique dont l'auctoritas a été assurée par l'invocation divine du proemium; mais une nouvelle fois, c'est l'identification avec l'octavien des guerres civiles que recouvre le terme deus $^{76}$. Nous pensons qu'ici Ovide articule la 
représentation virgilienne du deus pacificateur à la représentation horatienne du mythe de l'âge d'or; par l'articulation de ces deux héritages, le sens profond de la représentation divine est transformé, et offre ainsi une réponse aux nouvelles problématiques de l'époque augustéenne : si, par la réalité cultuelle du temps, Auguste peut bien se réclamer deus, il ne peut en revanche vouloir s'assimiler au Jupiter de la mythologie, sous peine de rompre l'illusion du retour de l'âge d'or et au risque d'une rupture de la pax deorum civile. Conscient du rôle que la mythologie poétique a joué dans l'avènement d'Octavien, et de l'exploitation postérieure qu'en a faite Auguste, Ovide chercherait-il à rétablir la séparation entre théologie civile et théologie mythique?

\section{BIBLIOGRAPHIE}

\section{Textes anciens}

Saint Augustin, Confessions. II, Livres IX-XIII, texte établi et trad. par P. DE LABRIOLLE, CUF, Paris, Les Belles Lettres, 1994.

Horace, Odes et Épodes, texte établi et trad. par F. VILLENEUVE, tirage revu par J. HELLEGOUARC'H, CUF, Paris, Les Belles Lettres, 2002.

Ovide, Les Métamorphoses. I, Livres I-IV, texte établi et trad. par G. LAFAYE, CUF, Paris, Les Belles Lettres, 2002.

Vergil, Eclogues, ed. by R. coleman, Cambridge Greek and Latin Classics, Cambridge, Cambridge University Press, 1989.

Virgile, Bucoliques, texte établi et trad. par E. DE SAINT-DENIS, introd. et notes de J.P. NÉRAUDAU, Paris, Les Belles Lettres, 2006.

Virgile, Bucoliques, texte établi par E. DE SAINT-DENIS ; trad. d'A. VIDEAU ; introd., commentaire et annotations d'H. CASANOVA-ROBIN, Commentario 2, Paris, Les Belles Lettres, 2014.

Virgile, Géorgiques, texte établi et trad. par E. DE SAINT-DENIS, CUF, Paris, Les Belles Lettres, 1982.

\section{Études modernes}

\section{Histoire politique et religieuse}

ANDRÉ J.-M. 1974, Le siècle d'Auguste, Le Regard de l'histoire, Paris, Payot.

DEONNA W. 1921, « La légende d'Octave-Auguste dieu, sauveur et maître du monde », RHR 83, p. 32-58.

ELIADE M. 1949, Traité d'histoire des religions, Bibliothèque scientifique, Paris, Payot. 
ÉTIENNE R. 1958, Le culte impérial dans la péninsule ibérique d'Auguste à Dioclétien, BEFAR 191, Paris, De Boccard, en particulier p. 288 (Livre III : «L'objet du culte ») à 403 (Livre IV : « L'évolution du culte impérial d'Auguste à Dioclétien »).

GAGÉ J. 1955, Apollon romain. Essai sur le culte d'Apollon et le développement du "ritus graecus" à Rome des origines à Auguste, BEFAR 182, Paris, De Boccard.

GRADEL I. 2002, Emperor Worship and Roman Religion, The Oxford Classical Monographs, Oxford, Clarendon Press.

HURLET F. 2015, « Devenir un dieu. La mort d'Auguste et la naissance de la monarchie impériale », in Augusto y el imperio romano, Studia historica. Historia antigua 32, Salamanque, Ed. Universidad de Salamanca, p. 61-75.

JAL P. 1961, « La propagande religieuse à Rome au cours des guerres civiles de la fin de la République », AC 30, 2, p. 395-414 [en ligne]. URL : http://www.persee.fr/doc/ antiq_0770-2817_1961_num_30_2_3414 [consulté le 4 janvier 2018] ; DOI : 10.3406/antiq. 1961.3414.

LA ROCCA E. 2011, « Dal culto di Ottaviano all' apoteosi di Augusto », in G. URSO (a cura di), Dicere laudes. Elogio, comunicazione, creazione del consenso. Atti del convegno internazionale, Cividale del Friuli, 23-25 settembre 2010, I convegni della Fondazione Niccolò Canussio 10, Pise, ETS, p. 179-204.

LAIGNOUX R. 2011, «L'utilisation de la religion dans la légitimation du pouvoir : quelques pistes de recherche pour les années 44-42 av. J.-C. », Cahiers Mondes anciens 2 [en ligne]. URL : https:// journals.openedition.org/mondesanciens/360 [consulté le 4 janvier 2018] ; DOI : 10.4000/ mondesanciens.360.

SAURON G. 2000, L'histoire végétalisée. Ornement et politique à Rome, Antiqua, Paris, Picard. SCHEID J. 1985, Religion et piété à Rome, Paris, Éd. de la découverte.

-2001, Religion et piété à Rome, Sciences des religions, Paris, A. Michel.

-2001-2002, « Religion, institutions et société de la Rome antique » [en ligne]. URL : https:// www.college-de-france.fr/media/john-scheid/UPL18440_38.pdf [consulté le 4 janvier 2018]. - 2004, « Comprendre le culte impérial. Autour de deux livres récents », AC 73, p. 239-249 [en ligne]. URL : http://www.persee.fr/doc/antiq_0770-2817_2004_num_73_1_2549 [consulté le 4 janvier 2018].

- 2006-2007, « Sacrifier pour l'Empereur, sacrifier à l'Empereur. Le culte des Empereurs sous le Haut-Empire romain » [en ligne]. URL : https://www.college-de-france.fr/media/john-scheid/ UPL18440_38.pdf [cours en ligne sur le site du Collège de France ; consulté le 16 août 2016]

\section{Littérature}

BAYET J. 1996, Littérature latine, $9^{\mathrm{e}}$ éd. revue et augm., Coll. U 266, Paris, A. Colin.

LE DOZE P. 2014, Le Parnasse face à l'Olympe. Poésie et culture politique à l'époque d'Octavien/Auguste, Coll. de l'École française de Rome 484, Rome, École française de Rome.

LEDENTU M. 2012, L'écrivain et le pouvoir : le fait littéraire et le fait politique à Rome (II siècle av. J.-C. - II siècle apr. J.-C.), Paris, Sorbonne IV, HDR non publiée.

PERNOT L. 1993, La rhétorique de l'éloge dans le monde gréco-romain, Coll. des études augustiniennes. Série Antiquité 137, Paris, Institut d'études augustiniennes, 2 vol. 


\section{Horace}

DELIGNON B., LE MEUR N., THÉVENAZ O. (dir.) 2016, La poésie lyrique dans la cité antique. Les Odes d'Horace au miroir de la lyrique grecque archaïque. Actes du colloque organisé les 6-8 juin 2012 par l'ENS de Lyon, Hisoma, UMR 5189, et l'Université de Lausanne, Lyon, Coll. du CEROR 49, Lyon, De Boccard.

FITZGERALD W. 2009, «Power and Impotence in Horace's Epodes », in M. LOWRIE (ed.), Horace: Odes and Epodes, Oxford Readings in Classical Studies, Oxford, Oxford University Press, p. 141-159. GRIFFIN J. 1997, «Cult and Personality in Horace », JRS 87, p. 54-69 [en ligne]. URL : http:// www.jstor.org/stable/301368 [consulté le 4 janvier 2018].

HEUZÉ P. 1997, «Quelques évidences vraies ou fausses chez Horace et Virgile », in C. LÉvY, L. PERNOT (éd.), Dire l'évidence. Philosophie et rhétorique antiques, Cahiers de philosophie ancienne et du langage de l'Université de Paris XII-Val-de-Marne 2, Paris, L'Harmattan, p. 197-206.

HILLS P. 2005, Horace, Ancients in Action, Londres, Bristol Classical Press.

LOWRIE M. (ed.) 2009, Horace: Odes and Epodes, Oxford Readings in Classical Studies, Oxford, Oxford University Press.

MCNEILL R. L. B. 2001, Horace: Image, Identity, and Audience, Baltimore, Johns Hopkins University Press.

MULROY D. 1994, Horace's Odes and Epodes, Ann Arbor, University of Michigan Press.

STARR C. G. 1969, « Horace and Augustus », AJPh 90, 1, p. 58-64 [en ligne]. URL : http://

www.jstor.org/stable/293304 [consulté le 4 janvier 2018].

VoISIN D. 2011, « Horace ou le refus de la poésie grecque courtisane », Rursus 6 [en ligne]. URL : https://journals.openedition.org/rursus/465 [consulté le 4 janvier 2018] ; DOI : 10.4000/rursus. 465.

WATSON L. C. 2003, A Commentary on Horace's Epodes, New York, Oxford University Press, 2003, notamment p. 479-533.

\section{Virgile}

BAILEY C. 1969, Religion in Virgil, New York, Barnes \& Noble.

BEAUJEU J. 1982, «L'enfant sans nom de la IV Be Bucolique », REL 60, p. 186-215.

BING P. 2016, « Epicurus and the iuuenis at Virgil's Eclogue 1.42 », CQ 66, 1, p. 172-179 [en ligne]. URL : https://doi.org/10.1017/S0009838816000173 [consulté le 4 janvier 2018] ; DOI : 10.1017/ S0009838816000173.

BRISSON J.-P. 1980, Virgile, son temps et le nôtre, $2^{\mathrm{e}}$ éd., Les Textes à l'appui, Paris, F. Maspero.

- 1992, Rome et l'âge d'or. De Catulle à Ovide, vie et mort d'un mythe, Textes à l'appui. Histoire classique, Paris, Éd. de la Découverte.

CARCOPINO J. 1943, Virgile et le mystère de la IV églogue, éd. revue et augm., Paris, L’Artisan du Livre. DELLA CORTE F. 1986, Le Georgiche di Virgilio. Commentate e tradotte, Pubblicazioni dell'Istituto di filologia classica e medievale 97-98, Gênes, Istituto di filologia classica e medievale, 2 vol. HORSFALl N. (ed.) 1995, A Companion to the Study of Virgil, Mnemosyne. Suppl. 151, Leyde, Brill. 
LEDENTU M. 2014, « Labor poétique et Res Gestae Caesaris : poésie et refondation dans les Géorgiques », VL 189-190, p. 70-88.

LEE M. O. 1996, Virgil as Orpheus. A Study of the Georgics, SUNY Series in Classical Studies, Albany, State University of New York Press.

SMITH R. A. 2011, Virgil, Blackwell Introductions to the Classical World, Malden, Wiley-Blackwell.

\section{Autres auteurs}

FABRE-SERRIS J. 1995, Mythe et poésie dans les Métamorphoses d'Ovide, Études et commentaires 104, Paris, Klincksieck.

LEHMANN Y. 1997, Varron théologien et philosophe romain, Coll. Latomus 237, Bruxelles, Latomus.

MINEO B. 2006, Tite-Live et l'histoire de Rome, Études et commentaires 107, Paris, Klincksieck.

VIDEAU A. 2010, La poétique d'Ovide, de l'élégie à l'épopée des Métamorphoses. Essai sur un style dans l'Histoire, Rome et ses renaissances, Paris, PUPS.

\section{NOTES}

1. GRIFFIN 1997, p. 54 : «It was the main contention of a book published a few years ago that the material and the attitudes which we find in the works of the Augustan poets are intimately connected with the realities of Roman life. [...] It was argued there that 'the same material can be observed at different levels of stylisation in different poetical contexts', and that we can learn a great deal about the poems and their authors by following the ways in which they employ and vary the same subject matter. [...] Those ideas are carried further in this paper, which extends that approach to another area: that of religious cult ».

2. La notion de héros ou de dieu salvateur est complexe à comprendre. On pourra néanmoins se référer à B. Mineo (2006, p. 137-154), qui définit les duces fatales comme des hommes providentiels, capables de faire régner la pax deorum et la concordia nationale, ainsi qu'à W. Deonna (1921), qui relève les principales caractéristiques des « maîtres du monde », perçus ou revendiqués comme « dieux sauveurs ». Parmi cellesci, le lien qu'il établit entre dieu sauveur et pacification de la nature, p. 52 sq., nous permet de comprendre le fleurissement du mythe de l'âge d'or à l'époque triumvirale.

3. Voir notamment SCHEID 1985 et SCHEID 2004, p. 245 : «La question n'est pas si l'individu était perçu comme dieu ou non. Ces honneurs exprimaient uniquement une relation entre l'honoré et celui qui l'honorait, la question du statut divin n'était pas posée. Elle n'a été posée qu'au moment de la christianisation, quand un Dieu absolu et universel revendique l'exclusivité de ces hommages ».

4. Voir scheID 2006-2007, p. 4 : «Il est inutile de se poser des questions comme celle de la croyance : les Romains et les Grecs croyaient-ils que l'empereur romain était un dieu, ou devenait dieu par l'apothéose ? C'est une question absurde, car comme les Anciens, il faut simplement réfléchir sur le processus rituel qui faisait d'un humain un diuus ».

5. Voir en particulier JAL 1961 et LAIGNOUX 2011. 
6. Voir E. La Rocca (2011, p. 181) notamment, qui récuse une datation tardive d'un culte à Octavien en Italie même : «Anche in Italia non mancavano forme di culto già un età precoce».

7. Pour une synthèse brève mais efficace des années 44-40 av. J.-C., voir BRISSON 1980, p. 81-89.

8. JAL 1961, p. 410. À noter cependant qu'il relève cette caractéristique pour les deux guerres civiles du $\mathrm{I}^{\mathrm{er}}$ s. av. J.-C.

9. Voir DEONNA 1921, p. 42, qui met en lumière le lien qui s'établit entre prétention à l'hégémonie sur terre et revendications divines.

10. LE DOZE 2014, p. 19-38 (et en particulier p. 28-38).

11. LE DOZE 2014, p. 36-37.

12. JAL 1961, p. 411.

13. Varron, dans ses Antiquités divines dont il ne reste aujourd'hui que des fragments, distinguait en effet la théologie civile, pratiquée dans le cadre de la cité, la théologie mythique, narrée par les poètes, et la théologie physique, qui concernait les philosophes.

14. Pour une analyse des rapports entre littérature grecque et littérature latine, voir notamment BAYET 1996, p. 198 sq., et CASANOVA-ROBIN (éd.) 2014, p. XI sq.

15. Voir I. Gradel (2002, p. 7), qui distingue genius et deus, le premier étant nécessairement la part divine de quelqu'un tandis que le terme deus désigne de manière absolue un dieu. À noter cependant le flottement terminologique qui règne chez les Anciens, et que reconnaît I. Gradel, p. 65-66, à propos de la distinction entre diuus et deus.

16. Bien que, comme souvent, la datation soit sujette à caution, on considère généralement que la rédaction des Bucoliques commença vers $42 \mathrm{av}$. J.-C. et que leur publication eut lieu en 39 av. J.-C. : voir notamment LEDENTU 2012, p. 35.

17. HEUZÉ 1997, p. 203. P. Heuzé explique d'ailleurs bien que la manière la plus sûre de créer de l'évidence sur une identité, c'est de donner le nom, ce que Virgile se refuse précisément à faire ici.

18. Pour un état de la question, qui fait encore débat, voir HORSFALL (ed.) 1995, p. 11-13, qui montre que les études récentes tendent à réduire la part d'autobiographie, mais non de réalité historique, dont on pourrait trouver la trace dans la première Églogue de Virgile.

19. Voir notamment BRISSON 1980, COLEMAN (éd.) 1989, HORSFALL (ed.) 1995, ou encore CASANOVA-ROBIN (éd.) 2014.

20. Voir cependant P. BING (2016, p. 173), qui rappelle que la mention équivoque d'un iuuenis ne peut faire trancher unanimement en faveur d'Octavien, bien que l'identification soit probable: la reprise du terme iuuenis en G., I, 500, pour désigner explicitement Octavien, ne peut être qu'intentionnelle. Mais selon lui, l'important n'est pas tant la figure à identifier que le fait que «Virgile laisse l'identité de cette figure incertaine ", ce qui a une fonction essentielle dans l'économie de la première Églogue.

21. Voir CASANOVA-ROBIN (éd.) 2014, p. 16-17, et LA ROCCA 2011, p. 182 : «è chiaro che in poesia l'assimilazione del principe a un dio ricalca formulazioni retoriche che avevano $\mathrm{i}$ 
loro precedenti nell' ambiente delle corti greco-ellenistiche», qui analyse cependant l'époque du Principat.

22. Virgile, Ecl., IV, 15-16 : Ille deum uitam accipiet diuisque uidebit / permixtos heroas et ipse uidebitur illis, «Il recevra une vie de dieux, verra les héros se mêler aux divinités et sera également vu parmi elles". La nature divine exacte de cet enfant reste cependant problématique, notamment à cause de l'imprécision du génitif poétique deum, dont nous avons tenté de rendre l'ambiguïté. Nous aurons à revenir sur cette question.

23. L'expression est de M. Ledentu, qui l'utilise à propos de la VIII Églogue.

24. Pour le travail virgilien de ce mythe, voir BRISSON 1980, p. 114-120.

25. Ce renvoi à la réalité de l'époque contemporaine se fait explicitement par la mention de Pollion: les vers 11 et 12, par l'insertion du nom du consul et l'ablatif absolu te consule, permettent d'expliciter à la fois le dédicataire du poème et le temps historique auquel se réfère Virgile.

26. On sait l'importance que prendra par la suite le mythe de l'âge d'or pour le Prince. La mention d'Apollon comme patron divin de cette nouvelle ère au vers 10 ne peut que nous rappeler la protection privilégiée d'Apollon dont Octavien prétendra par la suite bénéficier. Les vers 26-27, qui font mention de la lecture que fera le jeune enfant des exploits de son père, exempla à imiter voire à surpasser, développent un topos abondamment traité dans la littérature postérieure. Enfin, les vers 34-35, annonçant une nouvelle Argo et une seconde grande guerre, pourraient exprimer le pressentiment anticipé de la bataille d'Actium.

27. La somme d'analyses critiques sur ce passage est pléthorique : pour un état assez récent de la question, on consultera utilement BEAUJEU 1982, qui fait le bilan des différentes propositions d'identification. Parmi les hypothèses débattues, il nous faut avouer que celle de CARCOPINO 1943, p. 155 sq., nous a paru particulièrement séduisante, bien qu'elle n'ait pas remporté l'adhésion; mais nous ne pouvons nous résoudre à ne voir dans le puer qu'une unique allusion au fils de Pollion, réduisant ainsi la IV É Élogue à une poésie circonstanciée et complaisante. Peut-être Virgile avait-il en tête le fils de Pollion lorsqu'il rédigea cette églogue dont le consul fut choisi comme dédicataire. Mais sa poésie a su dépasser la circonstance, et donner voix à toutes les espérances, toutes les incarnations. L'article de J. Beaujeu oppose également plusieurs restrictions convaincantes à la thèse de J. Carcopino (p. 188-189). Pour autant, nous ne nous rallions pas non plus à l'hypothèse de son article, qui fait du puer virgilien l'incarnation anonyme de toute la jeunesse aristocratique. Nous pensons qu'une nouvelle fois il s'agit de l'une des possibilités seulement.

28. BRISSON 1992, p. 80.

29. BRISSON 1992, p. 81.

30. BRISSON 1992, p. 83.

31. BRISSON 1992, p. 83.

32. Cette identification du puer avec le jeune Octave est aussi soutenue par J.-P. Brisson, dont nous ne partageons cependant pas la vision critique et par trop dichotomique, qui sépare politique et poétique (1992, p. 89-107); sans doute était-il trop tôt encore pour que Virgile cherchât à dénoncer les revendications d'Octave et à crypter son message afin d'éviter le courroux des hommes politiques (remarquons à ce propos que J.-P. Brisson se focalise sur Octave sans mentionner Antoine, ce qui nous semble être 
une reconstruction a posteriori, en contradiction avec l'incertitude qui dominait cette période sombre de l'Histoire).

33. J.-P. Néraudau (2006, p. 40) est sans doute l'un de ceux qui, ces dernières années, ont le mieux mis en valeur le potentiel de cette interprétation, bien qu'une fois encore il ne parvienne pas à renoncer au démon de l'identification: "l'association de la prophétie avec la vie d'un enfant a naturellement incité les commentateurs, dès l'Antiquité, à identifier cet enfant. La bibliographie sur ce sujet est sans fin, et il ressort de ses contradictions et de ses incertitudes qu'il est vraisemblable que Virgile a pensé au fils de Pollion, auquel il dédie manifestement la pièce, tout en restant assez vague pour donner à tout autre enfant la chance de représenter la génération qui vient au monde dans l'année $40 \%$. Voir, plus récemment et plus explicitement encore, LEDENTU 2012, p. 45 : «La divergence des interprétations politiques que ce poème a suscitées correspond en fait à l'ambiguïté voulue par Virgile qui, conformément à l'esthétique de la pastorale mise en place dans la première pièce du recueil, refuse de prendre parti et de choisir entre les chefs politiques ». La perspective que nous choisissons de défendre est cependant un peu différente, car il ne s'agit alors plus pour Virgile de refuser la prise de position politique, mais la possibilité d'une identification unique dans son ensemble, élargissant ainsi la problématique de ce passage aux références métalittéraires notamment.

34. Nous entendons par héros salvateur un personnage dont le développement et le destin personnels sont indissociables de ceux de sa collectivité. C'est bien le rôle qu'endosse le puer de la IVe Églogue : voir notamment J.-P. Brisson (1992, p. 86 et 95), qui affirme que de la croissance de l'enfant dépend le sort de sa communauté. Il a déjà été noté que l'enfant n'est pas l'auteur de l'âge d'or, mais simplement le signe de son avènement (voir en particulier CARCOPINO 1943 et BEAUJEU 1982). Pour autant, le lien indissoluble que le poème fixe entre l'âge d'or et le puer nous pousse à penser que ce dernier, sans en être l'auteur, y possède un rôle fondamental, impossible à déterminer précisément, suivant, une nouvelle fois, la logique d'une poétique de la «fausse évidence » virgilienne, propre à embrasser tous les espoirs.

35. LA ROCCA 2011, p. 182 : «In quanto produzioni a carattere non peculiarmente statale e rientranti in un genere specifico di ormai lunga tradizione, le apologie poetiche non sono affatto un documento ufficiale dell' epoca, ma rispecchiano, piuttosto, quello che poteva essere il sentire comune del popolo nei confronti del loro benefattore, secondo l'opinione che la 'tangibilità' di beneficti ottenuti rapidamente in vita per merito di un principe puo valere talvolta assai di piu della sostanziale 'indifferenza con la quale gli dei guardano tanto alle buone, quanto alle cattive azioni ».

36. Voir LAIGNOUX 2011.

37. La figuration de l'Ara Pacis est un bon exemple de cette tentative de récupération a posteriori de la représentation littéraire. Voir SAURON 2000, p. 50 : on trouve sur l'Ara Pacis « les promesses d'une vie exubérante avec le retour de l'âge d'or présidé par les fils des vainqueurs d'Actium opposées à la mort volontaire des deux derniers acteurs criminels de l'âge de fer vaincus à Actium ». La promesse d'un âge d'or présidé par deux pueri nous laisse penser à un surenchérissement augustéen vis-à-vis de la IV É Églogue. Voir aussi, en ce sens, CARCOPINO 1943, p. 196 : «Quand, en 31 av. J.-C., Octave fut devenu le souverain maître de l'Empire; lorsque, quatre années plus tard, il prétendit inaugurer, avec le régime qu'il fondait, le nouvel âge d'or, il voulut détourner, à son profit, le courant mystique auquel l'églogue avait puisé », et p. 198 : "Auguste veilla 
personnellement à confisquer les idées qui pouvaient le nimber aux yeux de ses sujets d'une auréole surnaturelle». J. Beaujeu (1982, p.191-192) note bien «l'accent augustéen » de la IVe Églogue, mais n'en donne aucune explication.

38. LA ROCCA 2011, p. 182: "A Roma, adeguatasi [sic] rapidamente, almeno nei piu importanti testi letterari, a queste forme apologetiche che individuavano nel benefattore dell' umanità un emissario degli dei, o dio egli stesso, sembrava impossibile, al contrario, procedere ad un culto ufficiale controllato dallo stato, con riti e sacerdozi specifici, per l'innata ritrosia di un senato che non vedeva di buon occhio il predominio continuativo e duraturo di un magistrato sull' intera classe dirigente, se non in determinate occasioni sapientemente vincolate ».

39. Ce qui est la définition du héros salvateur, privilégié des dieux, mais n'en fait pas nécessairement un être divin, voir LA ROCCA 2011, p. 179-180.

40. FITZGERALD 2009, p. 146 : «Above all, though, it is the concern with the role of the poet in a time of political upheaval that makes the Epodes a successor to the Eglogues ».

41. La VII ${ }^{\mathrm{e}}$ Épode d'Horace, contemporaine de la XVI ${ }^{\mathrm{e}}$, mériterait également d'être étudiée. Par manque de place, nous avons préféré nous focaliser sur la seconde, la plus longue et la plus riche selon nous. L'Épode VII présente une réflexion similaire à celle qui nous occupera; il convient cependant de noter l'absence de mythe salutaire, comme si, dans ce texte, Horace n'espérait plus aucune échappatoire au sort contemporain de Rome.

42. Nous indiquons dans nos titres les dates supposées de rédaction. Ce sont en effet celles qui nous intéressent car elles sont au plus près des ressentis contemporains des événements qu'elles retranscrivent - ou au contraire refusent de retranscrire. La date de parution des œuvres est également importante mais n'implique pas la même analyse : elle permet d'observer le regard rétrospectif du poète sur son œuvre, prenant acte de nouveaux faits. Ce que nous cherchons à comprendre, pour notre part, est le rôle, volontaire et immédiat, de la littérature dans la propagande de l'époque et sa capacité à retranscrire les espoirs du moment. La date de l'Épode XVI est, encore aujourd'hui, l'objet de nombreux débats. La question principale est celle de son antériorité ou non par rapport à l'Églogue IV de Virgile, avec laquelle elle entre, de l'avis de tous, en discussion. Pour un état récent de la question, voir WaTson 2003, p. 486-488. Nous nous rattachons ici à l'argumentation qu'elle propose pour la datation de l'épode. La question de la chronologie relative entre l'Églogue IV et l'Épode XVI n'est cependant pas pour nous prioritaire; il nous importe avant tout de savoir que les deux œuvres sont contemporaines, afin d'analyser la manière dont les deux poètes ont respectivement répondu aux mêmes événements.

43. Les termes altera ... aetas et Roma, placés respectivement dès les vers 1 et 2 , sont un autre indice de la dimension nationale de ce carmen. Voir aussi FITZGERALD 2009, p. 143 : "The penultimate poem in the collection is the most Archilochean at leats in its pretensions to the kind of public role we associate with Archilochus' political poems ». C'est la place du je individuel en tant que voix communautaire, et la prédominance du blâme dans cette Épode, qui en font la digne héritière de la poésie hymnique et iambique grecque, legs récemment souligné par DELIGNON, LE MEUR, THÉVENAZ (dir.) 2016, à propos des Odes. M. Ledentu (2012, p. 53) a cependant déjà relevé l'héritage iambique qu'assume Horace dès la période de rédaction de l'Épode XVI : « Aussi l'utopie de la fuite vers les îles Fortunées et la peinture de l'âge d'or, qui occupent toute la seconde partie de l'épode, servent-elles en quelque sorte de contrepoids à la dimension publique et 
civique de la parole iambique de la première partie : elles l'orientent, dans un dialogue intertextuel avec les Bucoliques de Virgile, vers un ailleurs situé en dehors de la cité ».

44. V. 14.

45. Voir notamment J.-P. Brisson (1992, p. 7-8), qui énumère les caractéristiques suivantes: ignorance des horreurs de la guerre et des dangers de la navigation maritime, harmonie avec les dieux, raffinement des mets, absence d'agression des bêtes fauves ainsi que spontanéité et abondance de la nature.

46. Une autre originalité du mythe de l'âge d'or dans le poème horatien est, comme J.P. Brisson l'a déjà souligné (1992, p. 68), le déplacement du mythe vers un lointain non pas temporel mais géographique.

47. Cererem (v. 43) et rege (v. 56).

48. R. L. B. McNeil (2001, p. 120-121) note à juste titre l'absence totale de référence à Octavien, positive ou négative. Nous serions tentée de rajouter: l'absence totale de référence aux dirigeants de l'époque. Mais alors que R.L.B. McNeil voit dans cette absence une caractéristique de la jeunesse d'Horace, en retrait de la situation politique contemporaine, nous préférons y voir un rejet volontaire de cette politique, et donc une implication forte du poète dans les problématiques de son temps.

49. Voir WATSON 2003, p. 530.

50. Nous constatons que l'insistance d'Horace sur la pietas comme valeur salvatrice est antérieure à la propagande augustéenne, qui reprendra à son compte, une nouvelle fois, les représentations poétiques de l'époque des guerres civiles. Auguste avait donc bien compris qu'elles n'étaient pas seulement les propos de littéraires isolés, mais bien la retranscription des attentes de tout un peuple. L'antériorité de la poétique d'Horace sur la propagande augustéenne a récemment été soulignée, mais pour les écrits postérieurs du poète, les Odes essentiellement, voir DELIGNON, LE MEUR, THÉVENAZ (dir.) 2016, p. 9-10: "On a ainsi montré qu'il existe une interaction entre la poésie et la politique et que les Odes contribuent à la construction de l'idéologie augustéenne autant qu'elles s'en font l'écho ", et MINEO 2006, p.147-148: «c'est surtout dans le domaine religieux qu'Auguste voulut refonder la ville et lui permettre de connaître un nouveau cycle de protection grâce à une pietas qui devait gagner aux Romains une bienveillance divine qu'ils avaient perdue en négligeant le culte des dieux", et «J. M. André (Auguste, 1974, p. 122), estime que le souci d'expiation d'Auguste, après les guerres civiles, rend compte dans une large mesure des principales caractéristiques de la politique religieuse augustéenne. À l'appui de cette thèse, il cite une ode d'Horace (Odes, III, 6, 1,sq.) où apparaît précisément la nécessité de restaurer les temples, de mettre un terme à l'indifférence religieuse et de retrouver la faveur des dieux ».

51. Il nous semble cependant devoir minimiser la portée pessimiste qu'on lui a souvent accordée à cette époque : voir notamment BRISSON 1992, p. 67, et HILLS 2005, p. 26-27. Il y a certes un profond dégoût pour le temps présent; mais le refuge que propose Horace sur les îles des Bienheureux n'est pas selon nous un appel à l'abandon de Rome ; il s'agit plutôt d'un refus du jeu politique contemporain et d'un appel à l'unique possibilité de salut, le retour à la pietas.

52. Pour la datation des Géorgiques, I, voir notamment BRISSON 1980, p. 141.

53. Virgile, G., I, 121-146.

54. LEE 1996, p. 56 : «The great difference between this theodicy (125-145) and those in most mythologies is that here the father god ends the Golden Age and imposes work 
and hardship on man, not vindictively, as a punishement for some original sin, but providentially ».

55. Virgile, G., I, 121-124 : [...] Pater ipse colendi / haud facilem esse uiam uoluit primusque per artem / mouit agros, curis acuens mortalia corda / nec torpere graui passus sua regna ueterno, « Le Père des dieux lui-même a voulu rendre l'agriculture difficile ; le premier il a fait méthodiquement remuer les terres, en aiguisant par les soucis l'intelligence des mortels, et il n'a pas permis à son empire de s'engourdir dans une lourde torpeur " (trad. E. de Saint-Denis, 1982).

56. La reprise du terme iuuenis, cette fois pour caractériser Octavien, prouve bien que Virgile fait un retour sur son œuvre précédente et entend lui apporter une réponse différente : voir BING 2016, p. 173.

57. Virgile, G., I, 5-41 et 498-506.

58. LEE 1996, p. 64-66: "Virgil ends Book 1 as he began it, with a prayer that his country's gods spare the youthful Octavian, who comes to troubled Rome as a sōter a savior. [...] In short, while Book 1 is a classic portrayal of man stuggling [sic] with nature from his earliest days, forcing the earth to be productive and, in the course of his efforts, evolving into the most creative of all animals, it can also be seen as a portrayal, through nature's metaphor, of one gifted man struggling to bring order out of the chaos of history, of a man charged with an evolutionary mission, on his way to godhead. That is why, to preface and conclude the descriptions of work, there is a prayer ».

59. Virgile, G., I, 24-25: Tuque adeo, quem mox quae sint habitura deorum / concilia incertum est, urbisne inuisere, Caesar, "Et toi surtout, César, qu'accueilleront bientôt on ne sait quels conseils des dieux, regarderas-tu avec envie les villes? ", et 503-504: Iam pridem nobis caeli te regia, Caesar, / inuidet atque hominum queritur curare triumphos, «Depuis longtemps déjà, le céleste palais nous envie ta présence, César, et se plaint que tu prêtes attention à des triomphes humains ».

60. Virgile, G., III, 10-25.

61. DELLA CORTE 1986, p. 16.

62. Voir notamment LeE 1996, p. 80 ; HORSFALL (ed.) 1995, p. $72-73$; LEDENTU 2014, p. 84.

63. LEE 1996, p. 81.

64. SMITH 2011, p. 91: "Caesar is "in the middle" [...] both figuratively and, for the Georgics as a collection, literally ».

65. Virgile, G., III, 21-22 : ipse caput tonsae foliis ornatus oliuae / dona feram [...], «moimême, la tête ceinte de feuilles d'olivier coupées, je distribuerai les présents ».

66. Virgile, G., III, 9.

67. Nous remercions ici $\mathrm{M}^{\text {me }}$ Bénédicte Delignon, qui nous a aidée par ses commentaires, lors de ses cours de préparation à l'agrégation, à profiter de la richesse de ce proème.

68. M. Ledentu (2014, p. 79-87) a déjà souligné et analysé le lien inextricable qui, dans les Géorgiques, III, unit le poète et le politique dans la quête du $\mathrm{k} \lambda \varepsilon ́ o \zeta$.

69. Cette revendication du pouvoir poétique chez Horace a été récemment relevée, mais pour une période plus tardive, celle des Odes: voir DELIGNON, LE MEUR, THÉVENAZ (dir.) 2016, p. 16, «à Pindare, Horace emprunte l'affirmation du pouvoir poétique: seule la poésie peut assurer la renommée du laudandus; dire que ce dernier accédera à 
l'immortalité grâce à la poésie, c'est affirmer l'immortalité de la poésie elle-même, autrement dit la toute-puissance poétique ».

70. Nous rejoignons la pensée traditionnelle, qui place la rédaction de l'œuvre entre 2 av. J.-C. et 8 apr. J.-C.

71. Ovide, Met., I, 5-75.

72. Ovide, Met., I, 21 et 32.

73. Ovide, Met., I, 89-124

74. Ovide, Met., I, 113-115 : Postquam, Saturno tenebrosa in Tartara misso, / Sub Ioue mundus erat, subiit argentea proles, / auro deterior, fuluo pretiosior aere.

75. Ovide, Met., I, 57.

76. Le vocabulaire employé dans la cosmogonie renvoie nécessairement le lecteur au souvenir des guerres civiles : le combat des vents est qualifié de discordia fratrum au vers 60, tandis que le geste organisateur du démiurge est un écho direct à la propagande augustéenne: Ovide, Met. 24-25: quae postquam euoluit caecoque exemit aceruo, / dissociata locis concordi pace ligauit, « le dieu, après avoir démêlé les éléments et les avoir arrachés à la confusion de la masse, établit entre eux un lien de concorde et de paix, puisqu'ils étaient maintenant placés chacun en des endroits distincts ». Comme chez Virgile, on peut cependant imaginer d'autres identifications. Si J. Fabre-Serris (1995, p. 310) affirme en effet que «l'image du monde restauré après l'époque troublée des guerres civiles est symbolisée par le mythe de l'âge d'or », M. Eliade (1949, p. 319) rappelle quant à lui que «la cosmogonie est l'exemplaire type de toutes les constructions ».

\section{RÉSUMÉS}

Cet article propose une nouvelle interprétation de certains poèmes de Virgile et d'Horace interrogeant le mythe de l'âge d'or et son rapport à la divinité, en les reconnectant aux enjeux de leur époque, ceux de la guerre civile, afin d'analyser leur interaction avec la propagande religieuse des triumvirs. Les échos et le dialogue qui s'établissent entre les différents poèmes révèlent une constante de la poésie des guerres civiles : la dimension auto-réflexive des œuvres et leur ancrage sur la scène politique.

The reader will find here a new interpretation about some virgilian and horatian poems who wonder about the myth of the Golden Age and the place of the divinity in it, reconnecting them to the contemporaneous issues, those of the civil war, in order to analyse their interaction with the religious propaganda of the triumviri. The similarities and the dialogue established between the poems reveal a permanent feature of the civil war poetry: a self-reflexive dimension and a will to anchor the poetry in the politic sphere. 
INDEX

Keywords : Roman civil war, religious propaganda, deification, Augustus

Mots-clés : guerre civile à Rome, propagande religieuse, divinisation, Auguste

Index géographique : Rome

nomsmotscles Augustin, Horace, Ovide, Pindare, Varron, Virgile

\section{AUTEURS}

ALIÉNOR CARTOUX

UMR 5189 HiSoMA 